\title{
Yukawas and discrete symmetries in F-theory compactifications without section
}

\author{
Iñaki García-Etxebarria, Thomas W. Grimm and Jan Keitel \\ Max Planck Institute for Physics, \\ Föhringer Ring 6, 80805 Munich, Germany \\ E-mail: inaki@mpp.mpg.de, grimm@mpp.mpg.de, jkeitel@mpp.mpg.de
}

\begin{abstract}
In the case of F-theory compactifications on genus-one fibrations without section there are naturally appearing discrete symmetries, which we argue to be associated to geometrically massive U(1) gauge symmetries. These discrete symmetries are shown to induce non-trivial selection rules for the allowed Yukawa couplings in $\mathrm{SU}(N)$ gauge theories. The general discussion is exemplified using a concrete Calabi-Yau fourfold realizing an SU(5) GUT model. We observe that M2 instanton effects appear to play a key role in the generation of new superpotential terms and in the dynamics close to phase transition loci.
\end{abstract}

KeYwords: F-Theory, Superstring Vacua, String Duality

ARXIV EPRINT: 1408.6448 


\section{Contents}

1 Introduction 1

2 F-theory compactifications without section and Yukawa structures $\quad 3$

2.1 Physics of F-theory compactifications without section 3

$\begin{array}{lll}2.2 & \text { Discrete gauge symmetries } & 6\end{array}$

$\begin{array}{lll}2.3 & \text { Four-dimensional Yukawa structures } & 8\end{array}$

2.4 String interpretation of the Higgsing 9

3 A class of elliptic fibrations with discrete symmetries $\quad 12$

$\begin{array}{lll}3.1 & \text { Hypersurface equation in } \mathbb{P}_{112} & 12\end{array}$

$\begin{array}{lll}3.2 & \text { Non-Abelian matter curves and Yukawa points } & 13\end{array}$

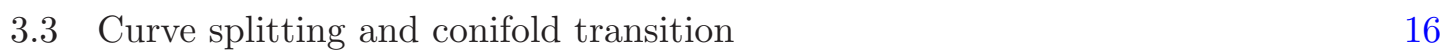

$\begin{array}{ll}3.4 & \text { Discrete charges and forbidden Yukawa couplings } \\ \end{array}$

$\begin{array}{ll}\text { 3.5 An explicit example without non-minimal singularities } & 17\end{array}$

$\begin{array}{llr}4 \text { Conclusions } & 18\end{array}$

\section{Introduction}

F-theory [1] compactifications to four dimensions are typically defined by specifying a $T^{2}$ fibered Calabi-Yau fourfold. The traditional assumption is that the fibration has a section, i.e. there is an embedding of the basis divisor into the total space, almost everywhere intersecting the fiber at a point. All such models are birational to a Weierstrass model [2]. Restricting oneself to Calabi-Yau fourfolds defined by Weierstrass models (and thus, having at least one section) simplifies model building with non-Abelian gauge symmetries, since there are well understood techniques for reading off the low energy non-abelian gauge groups from the structure of a Weierstrass model. ${ }^{1}$ Considerable effort has been made to develop similar techniques for analyzing and engineering elliptically fibered Calabi-Yau manifolds that also give rise to Abelian gauge groups in the low energy effective theory.

Initiated by the construction of the U(1)-restricted model in [4], the study of global F-theory compactifications with $\mathrm{U}(1)$ gauge factors can very roughly be divided into two approaches: (1) For a given U(1) gauge rank, one can determine the ambient space in which every elliptic fiber giving rise to such a low energy theory must be embeddable by using an old idea of Deligne [5]. Having obtained this space, one can then try to extract information about generic features of all such compactifications, such as all the matter representations can that possibly occur [6-11]. Non-generic elliptic fibers in Tate form

\footnotetext{
${ }^{1}$ See for example table 4 of [3] for a comprehensive dictionary between vanishing degrees of the Weierstrass model and the associated gauge algebras.
} 
were studied in $[12,13]$. (2) Conversely, one can take the stand and demand that given an arbitrary elliptically fibered Calabi-Yau manifold, one should be able to determine the low energy effective theory it gives rise to [14-16]. By breaking up the Calabi-Yau into its various building blocks and determining which of them can be treated separately, one can then systematically answer questions about entire classes of compactification manifolds [16] and find connections between them using Higgsings [20]. Alternatively, one could perform computer-aided scans over large numbers of compactifications as was done for example in $[21,22]$. Naturally, these two approaches are not mutually exclusive and there exist many ways in which they overlap. Additionally, work has been done to understand the geometry associated to singularities in higher codimensions in the base manifold [23] and the relations between the different ways of resolving these [24-26]. Furthermore, we note that a perpendicular approach has been taken by [27, 28], where resolutions are avoided by instead deforming the singularities, corresponding to a Higgsing of the gauge group.

By now, not only the Abelian gauge groups themselves, but also purely Abelian matter states, often called singlet states, appear to be fairly well understood in four and six dimensions, both from a geometric $[8,29,30]$ and a field theoretic perspective $[15,31,32]$ using the Chern-Simons terms of the effective theory compactified on a circle. Recently, a proposal for counting the precise number of multiplets in F-theory compactifications to four dimensions has been made [33]. In contrast, Yukawa couplings in global compactifications have been much less studied so far, both those that involve singlets and those that do not. While their assumed geometrical counterparts, intersections of different matter curves in codimension 3 in the base manifold, have received attention [7-10, 12, 20, 30, 34], it appears crucial to point out that the relation to T-branes [35, 36], and in particular the low energy effective theory and local models [37-42] remain to be explored.

Notably, beyond mathematical convenience there is no a priori physical reason to restrict oneself to $T^{2}$ fibrations with section. Calabi-Yau fourfolds with $T^{2}$ fiber but no section constitute perfectly respectable M-theory backgrounds, and they can admit a Ftheory limit. The physics of such backgrounds is rather interesting, and only recently it has been started to be systematically explored, mostly for the case of compactifications on threefolds [17-19]. ${ }^{2}$ In this paper we extend the physical picture put forward in [19] to Calabi-Yau fourfold compactifications without section. We propose a closed string and an open string perspective on the massive $\mathrm{U}(1)$ arising in compactifications without section, and comment on the geometrical configurations realizing this duality. Furthermore, we explicitly describe how a non-Abelian gauge theory on seven-branes can be engineered in such geometries. This allows us to argue that models without section do have potentially fruitful model building properties, such as the natural appearance of certain discrete symmetries at low energies. These discrete symmetries can (and do) forbid certain Yukawa couplings from being generated, even though the Yukawa couplings are otherwise allowed by all continuous symmetries present at low energies. Let us remark that intersecting D6 brane scenarios with similar physical implications have recently been studied for example in [45-47]. As we were completing this paper, [20] appeared in which discrete symmetries in F-theory compactifications are also studied.

\footnotetext{
${ }^{2}$ See also $[43,44]$ for earlier work on the topic.
} 
We start in section 2 with a quick review of some aspects of the physics of compactifications without section, where we explain the connection of discrete symmetries to certain geometrically massive U(1) symmetries, and we highlight the relevance of including M2 instanton effects in order to have a consistent description of the physics. In section 3 we then provide a detailed analysis of a phenomenologically motivated toy example, and show that indeed discrete symmetries forbid certain Yukawa couplings from appearing, in agreement with what the general discussion suggests.

\section{F-theory compactifications without section and Yukawa structures}

In this section we first discuss F-theory on genus-one fibrations without section generalizing the insights of [19] to Calabi-Yau fourfold compactification. This will be the first task of subsection 2.1, where we will provide two different perspectives, a closed string and an open string one, on massive $\mathrm{U}(1)$ gauge symmetries arising from such fibrations. Next, we examine the discrete symmetries that survive as remnants of the massive $\mathrm{U}(1)$ gauge symmetries at low energies in section 2.2. The Yukawa structures that arise in four-dimensional SU(5) Grand Unified Theories are treated in subsection 2.3, putting special emphasis on continuous and discrete selection rules. We also argue that an interesting class of instanton effects plays a key role in connecting the closed and the open string pictures. Finally, in subsection 2.4 we give a more detailed geometric description of the set-up and discuss the string interpretation of the Higgsing.

\subsection{Physics of F-theory compactifications without section}

In this section we first review the physics of F-theory compactifications on manifolds without section following the point of view taken in [19] (see also [17, 18, 20]). Next, it will be crucial to extend the discussion to a four-dimensional context, i.e. to the study of CalabiYau fourfolds without section.

Before turning to geometries without section, it is useful to first recall some facts about geometries with a section. In order that F-theory is well-defined, a potential Calabi-Yau compactification geometry should admit a genus-one fibration over some base manifold $B$. In this case the value of the dilaton-axion $\tau$, given by the complex structure modulus of the $T^{2}$ fiber, can be extracted from the geometry and describes a Type IIB string theory background. A subclass of such $T^{2}$ fibrations are geometries that have a section. The presence of a section implies the existence of a global meromorphic embedding of the base $B$ into the total space of the fibration. Alternatively, one can view a section as selecting precisely one point in the fiber over every point in the base with the possible exception of lower-dimensional loci in the base where the fiber degenerates. Fibrations with a section can be birationally transformed into a Weierstrass model given by

$$
y^{2}=x^{3}+f x z^{4}+g z^{6}
$$

where $(x: y: z)$ are the homogeneous coordinates of a $\mathbb{P}^{2,3,1}$, and $f, g$ are functions on $B$. A canonical section is simply given by $z=0$. While the F-theory literature has mostly 
focused on such Weierstrass models, the presence of a section is by no means a physical requirement for the existence of an effective F-theory action.

Let us thus turn to the case of having a genus-one fibered Calabi-Yau fourfold $\mathcal{X}_{4}$ without section. Despite the absence of a section such geometries still admit a multisection or $n$-section [17-20]. More precisely, while one cannot find a divisor cutting out a single point in the fiber over every point in the base, one can still find divisors singling out $n$ points in the fiber. These points may then undergo monodromies as one moves along the base $B$ of $\mathcal{X}_{4}$. The simplest case, which will be also the main focus in this work, is the situation where $n=2$, i.e. a manifold with a bi-section. It was argued in [19] that the effective action of F-theory compactified on such a manifold should include a massive $\mathrm{U}(1)$ gauge symmetry. In fact, one should rather think of the compactification as a cousin of a reduction with two sections, which would correspond to having an extra U(1) gauge symmetry present in the effective theory. Since the U(1) is massive in compactifications without section, let us recall that a $\mathrm{U}(1)$ can become massive by two related mechanisms: a linear Higgs mechanism or a non-linear Higgs mechanism, also known as the Stückelberg mechanism. It was argued in [19] that both points of view are useful to specify the effective theory obtained from a $\mathcal{X}_{4}$ compactification.

We start by describing the Stückelberg picture first. In this case the F-theory effective theory on $\mathcal{X}_{4}$ contains a $\mathrm{U}(1)$ that is massive due to the shift-gauging of an axion $c$ given by

$$
\hat{\mathcal{D}} c=d c+m \hat{A}^{1}, \quad c \rightarrow c-m \Lambda .
$$

Upon 'eating' the axionic degrees of freedom the kinetic term of $c$ turns into a mass term for $\hat{A}^{1}$. It was argued in [19] that for a geometry without section the axion involved in the gauging is a closed-string degree of freedom arising from the R-R or NS-NS two-form of Type IIB string theory. In other words, the geometries realize a geometrically massive $\mathrm{U}(1)$ gauge symmetry $[4,48,49]$. In fact, at weak string coupling $c$ is simply the zero-mode of the R-R two-form $C_{2}$ that renders a D7-brane $\mathrm{U}(1)$ massive [50].

Let us briefly recall the argument to justify that F-theory compactifications with a bi-section do indeed yield a Stückelberg massive U(1) in the effective theory. Following the suggestion of [51], it was shown in [19] that the M-theory to F-theory duality for such geometries requires the introduction of a background flux on the F-theory side. In order to connect M-theory and F-theory one has to consider the F-theory setup on an extra circle. Following the duality, the absence of a section requires to introduce circle flux $n$ along the extra circle. Indeed, at weak coupling this is due to a background flux for the field strength of the R-R two-form $C_{2}$. In the lower-dimensional theory the circle flux induces a further gauging

$$
\mathcal{D} c=d c+m A^{1}+n A^{0},
$$

where $A^{0}$ is the Kaluza-Klein vector. Taking into account that this implies the presence of a Stückelberg mass for the U(1) gauge field given by the linear combination $m A^{1}+n A^{0}$, it was shown that the M-theory and F-theory effective theories can indeed be matched. The presence of the Stückelberg gauging (2.2) coupling to the R-R or NS-NS two-form axion is crucial for this match to work. 
As pointed out above, the study of F-theory compactifications without section has so far focused on Calabi-Yau threefolds [17-20]. However, it is important to remark on how these considerations generalize to four-dimensional F-theory compactifications on CalabiYau fourfolds. In a four-dimensional theory with $\mathcal{N}=1$ supersymmetry the axion $c$ must arise from a complex field. We take it to be the real part of a complex field $G, \operatorname{Re} G=c$. The field $G$ is obtained when expanding the M-theory three-form as $[52,53]$

$$
C_{3}=i G \bar{\Psi}-i \bar{G} \Psi
$$

where $\Psi$ is a $(2,1)$-form on the Calabi-Yau fourfold $\mathcal{X}_{4}$. Using this definition of $G$, one can derive the four-dimensional effective theory. The relevant $\mathrm{U}(1)$ gauging appears in the kinetic term of $G$ given by

$$
\mathcal{L}_{4}=K_{G \bar{G}} \hat{\mathcal{D}}_{\mu} G \hat{\mathcal{D}}^{\mu} \bar{G}, \quad \hat{\mathcal{D}} G=d G+m \hat{A}^{1} .
$$

Upon 'eating' the axion $\operatorname{Re} G$, the kinetic term (2.5) becomes a mass term for $\hat{A}^{1}$, and the mass is simply given by $K_{G \bar{G}}$. Furthermore, it was shown in $[52,53]$ that for a massless $G$ $K_{G \bar{G}}$ takes the form

$$
K_{G \bar{G}}=\frac{i}{2 \mathcal{V}} \int_{\mathcal{X}_{4}} J \wedge \bar{\Psi} \wedge \Psi
$$

Note that since $\Psi$ is a $(2,1)$-form on $\mathcal{X}_{4}$, it depends on the complex structure moduli $z^{k}$ of $\mathcal{X}_{4}$. Remarkably, the moduli dependence of $\Psi$ can be specified by a holomorphic function $h(z)$. In the simplest situation one finds that $[53,54]$

$$
K_{G \bar{G}} \propto(\operatorname{Im} h)^{-1} .
$$

Moving along the complex structure moduli space, the coupling $K_{G \bar{G}}$ setting the mass of the $\mathrm{U}(1)$ can become zero.

Let us comment on the points at which the U(1) becomes massless. In order to do that, we extrapolate the behavior of $K_{G \bar{G}}$ using the results from a Calabi-Yau threefold. Indeed, the analog coupling in a Calabi-Yau threefold compactification depends crucially on the complex structure moduli and can be specified by a holomorphic pre-potential $\mathcal{F}(z)$. In this case, the function $h$ can be thought of as a second derivative of the pre-potential $\mathcal{F}(z)$. One then expects that at special points $z^{i} \approx 0, i=1, \ldots, n_{\text {con }}$ in complex structure moduli space one has

$$
h(z)=\sum_{i} a_{i} \log z^{i}+\ldots
$$

where $a_{i}$ are constants and the dots indicate terms that are polynomial in the complex structure parameter $z^{i}$. Geometrically, as we discuss in more detail below, this indicates that the points $z^{i}=0$ are conifold points and a geometric transition takes place. In fact, as discussed already in [19], the Calabi-Yau threefold with a bi-section $\mathcal{X}_{3}$ can transition to a Calabi-Yau threefold with two sections $\mathbb{X}_{3}$ by means of a conifold transition. In the 
Calabi-Yau fourfold case a similar transition from $\mathcal{X}_{4}$ to $\mathbb{X}_{4}$ can take place. In this case, however, one finds a whole curve of conifold points:

$$
\begin{array}{lllll}
\mathcal{X}_{3} & \text { tune } z^{i} & \mathcal{X}_{3}^{\text {sing }} \text { with conifold points } & \stackrel{\text { resolve }}{\longrightarrow} & \mathbb{X}_{3} \\
\mathcal{X}_{4} \stackrel{\text { tune } z^{i}}{\longrightarrow} & \mathcal{X}_{4}^{\text {sing }} \text { with conifold curve } & \text { resolve } & \mathbb{X}_{4}
\end{array}
$$

We stress that the resolved branch $\mathbb{X}$ can only be accessed in the lower-dimensional theory, i.e. in M-theory on $\mathbb{X}$. Nevertheless, the existence of the branch $\mathbb{X}$ naturally leads us to another interpretation of the setup with a U(1) made massive by a linear Higgs mechanism.

To introduce the linear Higgs mechanism picture, let us approach the singular geometry from the side of $\mathbb{X}_{4}$. At the singular point one also finds that there are new matter states in the four-dimensional effective theory, that are charged under the $\mathrm{U}(1)$. In other words, these admit the couplings

$$
\hat{\mathcal{D}} \phi=d \phi+i \hat{q} \hat{A}^{1} \phi
$$

where $\hat{q}$ is the $\mathrm{U}(1)$ charge of the complex field $\phi$. This implies that one can also think of giving a mass to the $\mathrm{U}(1)$ by turning on a vacuum expectation value (VEV) for the field $\phi$. In the F-theory compactifications under consideration the field $\phi$ will be a matter field arising from the open string sector on intersecting seven-branes. It will further be a singlet under the any additional non-Abelian group and therefore denoted by $\mathbf{1}_{\hat{q}}$, where the subscript indicates the U(1)-charge. Working with the open string matter field $\phi$ should be considered as the dual picture to working with the closed string field $G$. In order to match the charges one expects an identification

$$
\mathbf{1}_{\hat{q}} \text { (open string) } \leftrightarrow A(z) e^{2 \pi i r G} \text { (closed string), }
$$

where $m r=-\hat{q}$, and $A(z)$ is a coefficient that generally depends on the complex structure moduli of $\mathcal{X}_{4}$. Working with either $\mathbf{1}_{\hat{q}}$ or $G$ degrees of freedom should give a dual description of the same physical effective theory.

Let us close this section by noting that the fact that the $\mathrm{U}(1)$ is massive implies that it will be absent in the effective theory at energy scales below its mass. In this effective theory the selection rules originally imposed by the $U(1)$ gauge symmetry will remain as discrete symmetries. In the next section we therefore discuss discrete gauge symmetries of F-theory compactifications in more detail.

\subsection{Discrete gauge symmetries}

Let us now examine the Higgsing with respect to the discrete symmetries left over and use the restrictions that general F-theory spectra have to fulfill to our advantage. The set-up we consider consists of a $\mathrm{U}(1)_{0} \times \mathrm{U}(1)_{1} \times \mathrm{SU}(N)$ gauge group in the circle-compactified theory with matter states in the singlet, the fundamental $\mathbf{N}$ and the antisymmetric representation $\frac{1}{2} \mathbf{N}(\mathbf{N}-\mathbf{1})$ of $\mathrm{SU}(N)$. Here $\mathrm{U}(1)_{0}$ is the gauge group corresponding to the Kaluza-Klein vector and $\mathrm{U}(1)_{1}$ is the gauge factor lifting to the proper four-dimensional $\mathrm{U}(1)$ factor. Hence they correspond to the gauge fields $A^{0}$ and $A^{1}$ in (2.3) of the previous section. As 
noted for example in $[14,16]$, the allowed $\mathrm{U}(1)_{1}$ charges of all the occurring representations obey certain restrictions. First of all, let us assume that the $\mathrm{U}(1)_{1}$ generator has been rescaled such that the smallest singlet charge is $N$, thereby ensuring that all there are no fractional charges under $\mathrm{U}(1)_{1}$. Then the $\mathrm{U}(1)_{1}$ charges of the matter states fundamental representation satisfy

$$
Q_{\mathrm{U}(1)_{1}}(\mathbf{N}) \equiv k \quad \bmod N
$$

and the charges of the states in the antisymmetric representations fulfill

$$
Q_{\mathrm{U}(1)_{1}}\left(\frac{\mathbf{1}}{\mathbf{2}} \mathbf{N}(\mathbf{N}-\mathbf{1})\right) \equiv 2 k \bmod N,
$$

where $k$ is an integer defining the matter split [16] with respect to $\mathrm{U}(1)_{1}$. Let us now assume that a field in the $\mathbf{1}_{m, \hat{n} N}$ representation ${ }^{3}$ attains a VEV. For a general spectrum, the $\mathrm{U}(1)_{0} \times \mathrm{U}(1)_{1}$ symmetry is broken to $\mathrm{U}(1)^{\prime} \times\left(\mathbb{Z}_{m} \oplus \mathbb{Z}_{\hat{n} N}\right)$ while the $\mathrm{SU}(N)$ factor remains intact. Here $\mathrm{U}(1)^{\prime}$ is the linear combination $\hat{n} N \mathrm{U}(1)_{0}-m \mathrm{U}(1)_{1}$ under which the singlet with non-trivial VEV is uncharged. In terms of the old charges, the charges under the new gauge group are

$$
Q_{\mathrm{U}(1)^{\prime}}=\hat{n} N Q_{\mathrm{U}(1)_{0}}-m Q_{\mathrm{U}(1)_{1}}
$$

and

$$
Q_{\mathbb{Z}_{m}}=Q_{\mathrm{U}(1)_{0}} \quad \bmod m \quad Q_{\mathbb{Z}_{\hat{n} N}}=Q_{\mathrm{U}(1)_{1}} \quad \bmod \hat{n} N .
$$

Now let us be more specific and assume that the field Higgsing the U(1) gauge factor has charges $m=1$ and $\hat{n}=2$, as we find to be the case for all the models in which the elliptic fiber is embedded inside $\mathbb{P}_{112}$. Roughly speaking, this is due to the fact that states that are doubly charged under $\mathrm{U}(1)_{1}$ are intrinsically linked to states with non-trivial KK-charge, since the zero section also appears as a term in the divisor acting as the four-dimensional $\mathrm{U}(1)$ generator [19], and it is these states that appear at the conifold singularities. At first sight, the discrete gauge symmetry then appears to be $\mathbb{Z}_{2 N}$. However, we argue that it is in fact only $\mathbb{Z}_{2}$. To see this, look at all the possible charges of the matter representations:

$$
\begin{aligned}
Q_{\mathbb{Z}_{2 N}}(\mathbf{1}) & \in\{0, N\}, \\
Q_{\mathbb{Z}_{2 N}}(\mathbf{N}) & \in\{k, k+N\}, \quad Q_{\mathbb{Z}_{2 N}}\left(\frac{\mathbf{1}}{\mathbf{2}} \mathbf{N}(\mathbf{N}-\mathbf{1})\right) \in\{2 k, 2 k+N\} .
\end{aligned}
$$

Here we represent elements of $\mathbb{Z}_{2 N}$ by integers and the group law by addition modulo $2 N$. This implies

$$
\begin{aligned}
2 \cdot Q_{\mathbb{Z}_{2 N}}(\mathbf{1}) & =0, \\
2 \cdot Q_{\mathbb{Z}_{2 N}}(\mathbf{N}) & =2 k, \quad 2 \cdot Q_{\mathbb{Z}_{2 N}}\left(\frac{\mathbf{1}}{\mathbf{2}} \mathbf{N}(\mathbf{N}-\mathbf{1})\right)=4 k,
\end{aligned}
$$

\footnotetext{
${ }^{3}$ That is, the field transforms trivially under $\mathrm{SU}(N)$ and has charges $m$ and $\hat{n} \cdot N$ under $\mathrm{U}(1)_{0}$ and $\mathrm{U}(1)_{1}$, respectively. Note that $m$ here is the same as in (2.3), but we have chosen to write split up the $n$ from (2.3) into $n=\hat{n} \cdot N$ in order to emphasize that it contains a factor of $N$.
} 
which is an element of the center $\mathbb{Z}_{N}$ of the unbroken $\mathrm{SU}(N)$ factor. We therefore see that we can split $\mathbb{Z}_{2 N}$ into $\mathbb{Z}_{2} \oplus \mathbb{Z}_{N}$ and identify the second part with the center of the non-Abelian gauge group.

Finally, let us note that there are at least two kinds of special cases for which our discussion has to be adjusted. The first such case corresponds to a 0 -split, i.e. spectra of the above type with $k=0$. In this case all $\mathrm{U}(1)$ charges are divisible by $N$ and the second part of the discrete gauge group is $\mathbb{Z}_{\hat{n}}$ instead of $\mathbb{Z}_{\hat{n} N}$. Therefore the center of the $\operatorname{SU}(N)$ group drops out directly. The second case corresponds to set-ups where $N$ is even and $k=\frac{N}{2}$. In that case there may be additional identifications because fields that we treated independently above may be contained in the same multiplet.

\subsection{Four-dimensional Yukawa structures}

In the following we discuss the Yukawa structures of SU(5) GUTs engineered in an Ftheory compactification without section. Therefore, let us consider a SU(5) GUT with $\mathbf{1 0}$ representations and $\mathbf{5}$ representations. Furthermore, we include a number of GUT singlets 1. In order to make contact with the discussion of subsections 2.1 and 2.2 we distinguish representations by an additional $\mathrm{U}(1)_{1}$ charge, corresponding to the Abelian gauge field $\hat{A}^{1}$ introduced above. We indicate the $\mathrm{U}(1)_{1}$ charges of the $\mathbf{1 0}, \mathbf{5}$ and $\mathbf{1}$ states will by a subscript $q$ as in

$$
\mathbf{1 0}_{q}, \mathbf{5}_{q}, \mathbf{1}_{q}: \quad \mathbf{R}_{q} \rightarrow e^{2 \pi i q \Lambda} \mathbf{R}_{q},
$$

where a gauge transformation of $\hat{A}^{1}$ acts as $\hat{A}^{1} \rightarrow \hat{A}^{1}+d \Lambda$.

Since we are interested in Yukawa couplings, the relevant terms in the U(1)-invariant perturbative superpotential are

$$
W_{\text {pert }}: \quad \sum_{q_{1}+q_{2}+q_{3}=0} \mathbf{1 0}_{q_{1}} \mathbf{1 0}_{q_{2}} \boldsymbol{5}_{q_{3}}, \quad \sum_{q_{1}+q_{2}+q_{3}=0} \mathbf{1 0}_{q_{1}} \overline{\mathbf{s}}_{q_{2}} \overline{\mathbf{s}}_{q_{3}} .
$$

This generically implies that various couplings are absent. As an example, which we will realize in F-theory below, let us assume that we have a 4-split, i.e. $k=4$ in (2.13) and (2.14) with the representations

$$
\mathbf{5}_{-6}, \mathbf{5}_{-1}, \mathbf{5}_{4}, \mathbf{1 0}_{3}, \mathbf{1}_{5}, \mathbf{1}_{10} .
$$

The perturbatively permitted cubic Yukawas are then

$$
\mathbf{1 0}_{3} \times \mathbf{1 0}_{3} \times \mathbf{5}_{-6}, \quad \mathbf{1 0}_{3} \times \overline{\mathbf{5}}_{1} \times \overline{\mathbf{5}}_{-4},
$$

plus additional couplings involving the singlet states.

Let us now contrast this to the case in which the $\mathrm{U}(1)$ vector field has gained a mass term. As discussed above, this implies that the low-energy gauge symmetry is reduced to $\mathbb{Z}_{2} \times \mathrm{SU}(5)$. For our specific set-up we find that the $\mathbb{Z}_{2}$ charges are as follows:

$$
\begin{aligned}
& Q_{\mathbb{Z}_{2}}\left(\mathbf{5}_{4}\right)=0, \\
& Q_{\mathbb{Z}_{2}}\left(\boldsymbol{5}_{-1}\right)=1 \text {, } \\
& Q_{\mathbb{Z}_{2}}\left(\mathbf{5}_{-6}\right)=0, \\
& Q_{\mathbb{Z}_{2}}\left(\mathbf{1 0}_{3}\right)=1 \text {, } \\
& Q_{\mathbb{Z}_{2}}\left(\mathbf{1}_{5}\right)=1 \text {, } \\
& Q_{\mathbb{Z}_{2}}\left(\mathbf{1}_{10}\right)=0
\end{aligned}
$$


In particular, this means that at masses below the Stückelberg mass of our U(1) gauge field, the two curves $\mathbf{5}_{4}$ and $\mathbf{5}_{-6}$ should be indistinguishable. Furthermore, the singlets $\mathbf{1}_{10}$ are not charged under any massless gauge field anymore.

Under the remaining gauge symmetry, we expect to find the Yukawa couplings

$$
10_{3} \times 10_{3} \times \mathbf{5}_{-6}, \quad 10_{3} \times \mathbf{1 0}_{3} \times \mathbf{5}_{4}, \quad \mathbf{1 0}_{3} \times \overline{\mathbf{5}}_{1} \times \overline{\mathbf{5}}_{-4}, \quad \mathbf{1 0}_{3} \times \overline{\mathbf{5}}_{1} \times \overline{\mathbf{5}}_{6}
$$

plus additional couplings involving the singlet states. It is crucial to point out, however, that the coupling $\mathbf{1 0}_{3} \times \mathbf{1 0}_{3} \times \mathbf{5}_{-1}$ is still ruled out by the $\mathbb{Z}_{2}$ symmetry and we do not expect it to be realized in our example geometries.

It is particularly interesting to stress the role of the singlets in the setup. In the example of section 3 , we show that the singlet states $\mathbf{1}_{10}$ are involved in the Higgsing described in the previous subsection 2.1. In fact, the spectrum (2.21) arises in the open string interpretation of the F-theory setting. The closed string axion appears as the phase of the $\mathbf{1}_{10}$ using the identification (2.12). Furthermore, we will find in our concrete example that there are couplings of the form

$$
\mathbf{1}_{10} \times \mathbf{5}_{-6} \times \overline{\mathbf{5}}_{-4}
$$

Given such a coupling in the open string picture, one may thus wonder whether from the closed string point of view a non-perturbative superpotential appears that involves the complex field $G$. Concretely, inspired by the identification (2.12) we have in mind terms of the form

$$
W_{\text {non-pert }}=\cdots+\sum_{q_{1}+q_{2}-r m=0} A(z) e^{2 \pi i r G} \mathbf{5}_{q_{1}} \overline{\mathbf{s}}_{q_{2}} .
$$

As we will explain in subsection 2.4, some of these couplings are indeed present, and can be reinterpreted in terms of the classical couplings (2.25).

Let us close this subsection with some comments on the non-perturbative couplings (2.26). Superpotential couplings of a similar type induced by stringy instantons have been studied intensively in orientifold compactifications as reviewed in detail in [55]. Remarkably, the couplings (2.26) appear to be of somewhat different nature. They do not depend on the Kähler moduli and therefore are not suppressed at large volume. However, this is not a contradiction to a de-compactification argument, since these couplings are localized near the intersection of seven-branes. The instantons give a mass for certain $\mathbf{5}$ states that will therefore be absent in the effective theory for the massless modes only. We will see in our concrete examples that this picture is indeed consistent. It would be very interesting to perform a more thorough study of the instantons inducing the couplings (2.26). Interestingly, this can already be done in the weak coupling limit.

\subsection{String interpretation of the Higgsing}

Let us now try to understand better the link between geometric quantities on the one hand and field theory quantities on the other. We emphasize that the fact that a new branch of moduli space opens up in the M-theory compactification, connecting via a geometric 
transition our Calabi-Yau background to a large network of spaces, is not essential for our discussion. An alternative, more self-contained, viewpoint is that we are studying the physics of the Higgsed (i.e. deformed) branch close to a particular point in moduli space where extra degrees of freedom appear. Nevertheless, we will keep using the M-theory viewpoint for convenience, since discussions about geometry and M2-brane states can be easily understood there.

Let us start with the case of the five-dimensional transition, i.e. a conifold transition for a Calabi-Yau threefold in M-theory. This case is well understood by now and we briefly recall the discussion of the transition given in $[56,57]$. Take a Calabi-Yau threefold $\mathcal{X}$. As we tune some of the complex structure moduli, there are codimension $R$ subspaces in complex structure moduli space where $\mathcal{X}$ develops conifold singularities. Geometrically, this implies the simultaneous vanishing of a number of periods

$$
z^{i}=\int_{\Pi_{i}} \Omega, \quad i=1, \ldots, P
$$

with $\Pi_{i}$ a set of elements of $H^{3}(\mathcal{X}, \mathbb{Z})$, and $\Omega$ the holomorphic three-form of $\mathcal{X}$. More pictorially, we have $P$ 3-spheres contracting to zero size. Not all of these 3 -spheres are homologically independent, only $R$ of them are. Our examples all have $P-R=1$, and henceforth we restrict the discussion to this case for concreteness.

Consider the defining equation of the Calabi-Yau fourfold without a section that we will study later, which is of the form ${ }^{4}$

$$
\begin{aligned}
p_{112}= & \tilde{a}_{0} w^{2}+\tilde{a}_{1} y_{1}^{2} w+\tilde{a}_{2} y_{1} y_{2} w+\tilde{a}_{3} y_{2}^{2} w+\tilde{a}_{4} y_{1}^{4} \\
& +\tilde{a}_{5} y_{1}^{3} y_{2}+\tilde{a}_{6} y_{1}^{2} y_{2}^{2}+\tilde{a}_{7} y_{1} y_{2}^{3}+\tilde{a}_{8} y_{2}^{4} \\
= & 0,
\end{aligned}
$$

with the $\tilde{a}_{i}$ being sections of line bundles of appropriate degree in the base. The conifold locus in moduli space is obtained by tuning $R$ coefficients in this equation, which allow us to set $\tilde{a}_{8}=0$, modulo local coordinate redefinitions. An argument in [19] then shows that there are conifold singularities at the $P$ points in the base given by the solutions of $\tilde{a}_{3}=\tilde{a}_{7}=0$.

In the five-dimensional effective field theory, as we approach the conifold locus, a massive $\mathrm{U}(1)$ vector multiplet becomes light. When we hit the conifold locus in moduli space the massive vector multiplet becomes massless, and it splits into a massless vector multiplet and a massless charged hyper. The physics is thus that of an unHiggsing process. Going in the reverse direction, i.e. taking $\tilde{a}_{8} \neq 0$, corresponds to giving a VEV to the charged hyper, and thus an ordinary five-dimensional Higgsing process.

For our purposes it will be useful to understand the geometric manifestation of this Higgsing in more detail. (The basic picture was given in [58].) Consider the theory at the conifold locus. We have a massless $\mathrm{U}(1)$ vector multiplet, ${ }^{5}$ which in M-theory comes from

\footnotetext{
${ }^{4}$ We changed notation with respect to [19], the most relevant part of the dictionary for comparison to that paper is $\left\{\tilde{a}_{8}, \tilde{a}_{3}, \tilde{a}_{7}\right\} \rightarrow\{a, f, e\}$.

${ }^{5}$ Typically there will be other $\mathrm{U}(1)$ vector multiplets in the low energy theory, but one can choose a basis in which they decouple from the physics of the transition.
} 
a supergravity reduction of the form $C_{3}=A \wedge \omega$, with $A$ the five-dimensional vector boson and $\omega$ a harmonic two-form in the threefold $\mathcal{X}$. By Poincaré duality, we can also think of $\omega$ as defining a four-cycle $D$ in $\mathcal{X}$.

As we start making $\tilde{a}_{8} \neq 0$, the $\mathrm{U}(1)$ should acquire a mass. The geometric manifestation of this fact is that $\omega$ is no longer a harmonic form, but rather becomes a low-lying eigenform of the Laplacian of $\mathcal{X}$, or dually, the four-cycle $D$ becomes a four-chain with boundary. In fact, the four-chain is easy to describe: as we deform away from the conifold locus, the $P$ conifold singularities are replaced by $P$ three-spheres $S_{i}$. There is a relation in homology between these spheres, i.e. there is a four-chain in homology with boundary on these spheres. This four-cycle is $D$.

Coming back to the $\tilde{a}_{8}=0$ conifold locus, we have that there are also $P$ hypermultiplets charged under the U(1). They come from M2 branes wrapping the vanishing size holomorphic $S^{2}$ at the conifold singularity. As we deform away from the conifold locus, $R=P-1$ hypermultiplets stay massless, and get reinterpreted in the geometry as complex structure moduli of the $R$ growing classes in homology, plus the integrals of $C_{3}$ and $C_{6}$ over the same homology classes. The massive vector boson comes from reducing $C_{3}$ over the (non-zero) eigenform of the Laplacian connected to the four-cycle becoming a four-chain in the conifold transition. From this discussion, it follows that one should identify the closed string axion entering the Stückelberg mechanism in the geometric description of the massive U(1) given above with the phase of the charged hypermultiplet getting a VEV and entering the non-linear realization of the $\mathrm{U}(1)$ gauge symmetry becoming massive.

One take-home message from this discussion is that there is a deep interrelation between the field theory and the geometry, and a duality dictionary of sorts: what we see in the field theory as a Higgsing of a field appears in the geometry as a particular four-cycle getting boundaries and becoming a four-chain. There is also a nice interplay between field theory and string theory when it comes to the corrections to the theory: as explained in [58], and further substantiated in [59], in order to reproduce the right hypermultiplet moduli space metric one expects from field theory, one should sum an infinite set of nonperturbative corrections coming from M2 brane instantons in M-theory.

A similar picture will hold in the case of compactifications on a Calabi-Yau fourfold. We now have an M-theory compactification down to three dimensions, and there is a $\mathrm{U}(1)$ symmetry that becomes Higgsed as we resolve the conifold singularities. The U(1) vector boson comes from the reduction of $C_{3}=A \wedge \omega$. Poincaré duality now tells us that we should be looking for a six-cycle in the geometry that opens up in the resolution process and has boundaries on five-cycles. These five-cycles have a simple interpretation: instead of having conifold points in the total space, we now have conifold curves. As we deform the defining equation, we obtain a set of five-cycles given by fibrations of the deformation $S^{3}$ over the matter curve being Higgsed. ${ }^{6}$ The massive U(1) is associated with the open chain with boundaries on these five-cycles. The conifold periods analog to (2.27) can be studied using the recent results of $[61,62]$. However, the relevant couplings, as discussed in subsection 2.1, should rather be encoded by $J \wedge \Psi$ integrated over the five-cycles involved in the transition.

\footnotetext{
${ }^{6}$ Note that this kind of setup has been studied before in [60].
} 
We now obtain a possible reinterpretation of the perturbative field theory discussion in terms of geometry: the cubic terms that give rise upon Higgsing to mass couplings between the two 5 curves that recombine can be understood geometrically as being given by M2 instanton corrections wrapping the contracting three-cycle, as we approach the conifold point at $\tilde{a}_{8}=0$. Notice that the discussion is reminiscent of the $\mathcal{N}=2$ discussion in $[58,59]$. It would be quite interesting here, for the same reasons, to elucidate the microscopics of the instanton viewpoint.

\section{A class of elliptic fibrations with discrete symmetries}

In this section, we present a class of Calabi-Yau manifolds that realize the effects discussed in the preceding discussion. To do so, we start in subsection 3.1 by constructing a class of elliptically fibered manifolds without section, with fiber a generic quartic in $\mathbb{P}_{112}$. Next, we enforce an SU(5) singularity along a divisor of the base manifold and study the low-energy effective action of F-theory on the Calabi-Yau manifold. In section 3.2 we find that despite the absence of massless U(1) gauge factors in the effective action, there are different matter curves distinguished by a discrete gauge symmetry that is a remnant of a massive $\mathrm{U}(1)$ vector field. Furthermore, we encounter that not all the Yukawa couplings that would naively be allowed by the $\mathrm{SU}(5)$ gauge symmetry are realized geometrically. In fact, we show that those couplings that do exist correspond precisely to those invariant under the additional discrete symmetry.

Moving to the conifold locus in complex structure moduli space we note in section 3.3 that one of the matter curves becomes reducible and splits into two parts. We note that this is a manifestation of the $\mathrm{U}(1)$ becoming massless at the singular point and the restoration of the full Abelian gauge symmetry. Resolving the conifold singularities allows us to confirm that the map between the full $\mathrm{U}(1)$ charges and the charge under the discrete remnant group left over after the Higgsing process is as expected.

\subsection{Hypersurface equation in $\mathbb{P}_{112}$}

Following the discussion in [19], we embed a genus-one curve inside $\mathbb{P}_{112}$. The most general such genus-one curve is given by (2.28), which we reproduce here

$$
\begin{aligned}
p_{112}= & \tilde{a}_{0} w^{2}+\tilde{a}_{1} y_{1}^{2} w+\tilde{a}_{2} y_{1} y_{2} w+\tilde{a}_{3} y_{2}^{2} w \\
& \quad+\tilde{a}_{4} y_{1}^{4}+\tilde{a}_{5} y_{1}^{3} y_{2}+\tilde{a}_{6} y_{1}^{2} y_{2}^{2}+\tilde{a}_{7} y_{1} y_{2}^{3}+\tilde{a}_{8} y_{2}^{4} \\
=0, &
\end{aligned}
$$

where the $\tilde{a}_{i}$ determine the complex structure of the genus-one curve. After fibering the curve over a suitable base, the $\tilde{a}_{i}$ become sections of line bundles over the base manifold. As discussed in [19], an elliptic fibration with such a generic fiber does not have a section, but rather a two-section defined by $y_{1}=0$. However, after tuning $\tilde{a}_{8} \rightarrow 0$ the genus-one curve becomes singular and the two-section splits into two independent sections. These can then be most conveniently described after resolving the singularity obtained by the tuning. Note further that $\mathbb{P}_{112}$ exhibits an orbifold singularity at the origin and, in general, 
this singularity should be resolved. Here, however, we restrain from doing so and instead impose a condition on $\tilde{a}_{0}$ later on that makes sure that our hypersurface does not hit the orbifold singularity.

Next, let us tune the complex structure coefficients in such a manner that the elliptic fibration obtains an SU(5) singularity and then resolve this singularity using methods from toric geometry. In general, there are many inequivalent ways of creating such a singularity and then resolving it. Toric resolutions of such singularities were classified using the formalism of tops in [63] and, for the case of SU(5), evaluated explicitly in [16]. In the language of [16] the ambient fiber space $\mathbb{P}_{112}$ correspond to the polygon $F_{4}$ and there are three inequivalent tops. ${ }^{7}$ Let us pick the first one, called $\tau_{4,1}$ in [16], and denote the four blow-up variables and the variable corresponding to the affine node by $e_{i}, i=0, \ldots, 4$. Then this choice of SU(5) top implies that the coefficients $a_{i}$ must factor according to

$$
\begin{array}{llll}
\tilde{a}_{0}=e_{0}^{2} e_{1} e_{4} \cdot a_{0} & \tilde{a}_{1}=e_{1} e_{2} \cdot a_{1} & \tilde{a}_{3}=e_{0} e_{3} e_{4} \cdot a_{3} & \tilde{a}_{4}=e_{1}^{3} e_{2}^{4} e_{3}^{2} e_{4} \cdot a_{4} \\
\tilde{a}_{5}=e_{1}^{2} e_{2}^{3} e_{3}^{2} e_{4} \cdot a_{5} & \tilde{a}_{6}=e_{1} e_{2}^{2} e_{3}^{2} e_{4} \cdot a_{6} & \tilde{a}_{7}=e_{2} e_{3}^{2} e_{4} \cdot a_{7} & \tilde{a}_{8}=e_{0} e_{2} e_{3}^{3} e_{4}^{2} \cdot a_{8},
\end{array}
$$

where the $a_{i}$ are irreducible polynomials and $\tilde{a}_{2}=a_{2}$. Unlike the $\tilde{a}_{i}$, it is crucial that the $a_{i}$ depend on $e_{i}$ only through the combination $e_{0} e_{1} e_{2} e_{3} e_{4}$.

\subsection{Non-Abelian matter curves and Yukawa points}

Having tuned the complex structure coefficients in the above manner, the next step is to verify that this does produce an SU(5) singularity and to examine what sort of matter representations arise at codimension two in the base manifold.

To do this, let us now compute the Weierstrass form (2.1) of the Jacobian of the above genus-one curve. One finds that the Weierstrass coefficients $f$ and $g$ also depend on the $e_{i}$ only through the combination $e_{0} e_{1} e_{2} e_{3} e_{4}$ and we can therefore go to a patch in which $e_{1}=e_{2}=e_{3}=e_{4}=1$ without losing any information. In that case $f$ and $g$ read

$$
\begin{aligned}
f=-\frac{1}{48} \cdot\left(a_{2}^{4}-\right. & 8 e_{0} \cdot a_{1} \cdot a_{2}^{2} \cdot a_{3}+8 e_{0}^{2} \cdot\left(2 a_{1}^{2} a_{3}^{2}-a_{0} a_{2}^{2} a_{6}+3 a_{0} a_{1} a_{2} a_{7}\right) \\
& +8 e_{0}^{3} \cdot a_{0} \cdot\left(3 a_{2} a_{3} a_{5}-2 a_{1} a_{3} a_{6}-6 a_{1}^{2} a_{8}\right) \\
& \left.+16 e_{0}^{4} \cdot a_{0} \cdot\left(-3 a_{3}^{2} a_{4}+a_{0} a_{6}^{2}-3 a_{0} a_{5} a_{7}\right)+192 e_{0}^{5} \cdot a_{0}^{2} a_{4} a_{8}\right)
\end{aligned}
$$

and

$$
\begin{gathered}
g=\frac{1}{864} \cdot\left(a_{2}^{6}-12 e_{0} \cdot a_{1} \cdot a_{2}^{4} \cdot a_{3}+12 e_{0}^{2} \cdot a_{2}^{2} \cdot\left(4 a_{1}^{2} a_{3}^{2}-a_{0} a_{2}^{2} a_{6}+3 a_{0} a_{1} a_{2} a_{7}\right)\right. \\
+4 e_{0}^{3} \cdot\left(-16 a_{1}^{3} a_{3}^{3}+9 a_{0} a_{2}^{3} a_{3} a_{5}+6 a_{0} a_{1} a_{2}^{2} a_{3} a_{6}-36 a_{0} a_{1}^{2} a_{2} a_{3} a_{7}-18 a_{0} a_{1}^{2} a_{2}^{2} a_{8}\right) \\
+12 e_{0}^{4} \cdot a_{0} \cdot\left(-6 a_{2}^{2} a_{3}^{2} a_{4}-12 a_{1} a_{2} a_{3}^{2} a_{5}+8 a_{1}^{2} a_{3}^{2} a_{6}+4 a_{0} a_{2}^{2} a_{6}^{2}\right. \\
\left.-6 a_{0}^{2} a_{2}^{2} a_{5} a_{7}-12 a_{0}^{2} a_{1} a_{2} a_{6} a_{7}+18 a_{0}^{2} a_{1}^{2} a_{7}^{2}+24 a_{0} a_{1}^{3} a_{3} a_{8}\right)
\end{gathered}
$$

\footnotetext{
${ }^{7}$ Put differently, that means that there are three different ways of engineering a resolved SU(5) singularity. Note that one of the tops, called $\tau_{4,2}$ in [16], leads to non-minimal singularities even for Calabi-Yau threefolds.
} 


$$
\begin{aligned}
& +48 e_{0}^{5} \cdot a_{0} \cdot\left(6 a_{1} a_{3}^{3} a_{4}-3 a_{0} a_{2} a_{3} a_{5} a_{6}+2 a_{0} a_{1} a_{3} a_{6}^{2}+18 a_{0} a_{2} a_{3} a_{4} a_{7}\right. \\
& \left.\quad-3 a_{0} a_{1} a_{3} a_{5} a_{7}-12 a_{0} a_{2}^{2} a_{4} a_{8}+18 a_{0} a_{1} a_{2} a_{5} a_{8}-12 a_{0} a_{1}^{2} a_{6} a_{8}\right) \\
& +8 e_{0}^{6} \cdot a_{0}^{2} \cdot\left(27 a_{3}^{2} a_{5}^{2}-72 a_{3}^{2} a_{4} a_{6}-8 a_{0} a_{6}^{3}\right. \\
& \left.\quad+36 a_{0} a_{5} a_{6} a_{7}-108 a_{0} a_{4} a_{7}^{2}-144 a_{1} a_{3} a_{4} a_{8}\right) \\
& \left.+288 e_{0}^{7} \cdot a_{0}^{3} \cdot\left(-3 a_{5}^{2} a_{8}+8 a_{4} a_{6} a_{8}\right)\right) .
\end{aligned}
$$

From that it follows directly that the discriminant, defined by $\Delta=4 f^{3}+27 g^{2}$, obeys

$$
\begin{aligned}
& \Delta=\frac{a_{0}^{2}}{16} \cdot\left(e_{0}^{5} \cdot a_{2}^{4} \cdot\left(-a_{3} a_{7}+a_{2} a_{8}\right) \cdot\left(-a_{2}^{3} a_{4}+a_{1} a_{2}^{2} a_{5}-a_{1}^{2} a_{2} a_{6}+a_{1}^{3} a_{7}\right)\right. \\
& -e_{0}^{6} \cdot a_{2}^{2} \cdot\left(a_{2}^{4} a_{3}^{2} a_{4} a_{6}-a_{1} a_{2}^{3} a_{3}^{2} a_{5} a_{6}+a_{1}^{2} a_{2}^{2} a_{3}^{2} a_{6}^{2}+11 a_{1} a_{2}^{3} a_{3}^{2} a_{4} a_{7}\right. \\
& -10 a_{1}^{2} a_{2}^{2} a_{3}^{2} a_{5} a_{7}+8 a_{1}^{3} a_{2} a_{3}^{2} a_{6} a_{7}-8 a_{1}^{4} a_{3}^{2} a_{7}^{2}+a_{0} a_{2}^{4} a_{4} a_{7}^{2} \\
& -a_{0} a_{1} a_{2}^{3} a_{5} a_{7}^{2}+a_{0} a_{1}^{2} a_{2}^{2} a_{6} a_{7}^{2}-a_{0} a_{1}^{3} a_{2} a_{7}^{3}-12 a_{1} a_{2}^{4} a_{3} a_{4} a_{8} \\
& \left.+11 a_{1}^{2} a_{2}^{3} a_{3} a_{5} a_{8}-10 a_{1}^{3} a_{2}^{2} a_{3} a_{6} a_{8}+8 a_{1}^{4} a_{2} a_{3} a_{7} a_{8}+a_{1}^{4} a_{2}^{2} a_{8}^{2}\right) \\
& +e_{0}^{7} \cdot\left(a_{2}^{5} a_{3}^{3} a_{4} a_{5}-a_{1} a_{2}^{4} a_{3}^{3} a_{5}^{2}+10 a_{1} a_{2}^{4} a_{3}^{3} a_{4} a_{6}-8 a_{1}^{2} a_{2}^{3} a_{3}^{3} a_{5} a_{6}+8 a_{1}^{3} a_{2}^{2} a_{3}^{3} a_{6}^{2}\right. \\
& +40 a_{1}^{2} a_{2}^{3} a_{3}^{3} a_{4} a_{7}-32 a_{1}^{3} a_{2}^{2} a_{3}^{3} a_{5} a_{7}+a_{0} a_{2}^{5} a_{3} a_{5}^{2} a_{7}+16 a_{1}^{4} a_{2} a_{3}^{3} a_{6} a_{7} \\
& -12 a_{0} a_{2}^{5} a_{3} a_{4} a_{6} a_{7}+8 a_{0} a_{1} a_{2}^{4} a_{3} a_{5} a_{6} a_{7}-8 a_{0} a_{1}^{2} a_{2}^{3} a_{3} a_{6}^{2} a_{7}-16 a_{1}^{5} a_{3}^{3} a_{7}^{2} \\
& +48 a_{0} a_{1} a_{2}^{4} a_{3} a_{4} a_{7}^{2}-41 a_{0} a_{1}^{2} a_{2}^{3} a_{3} a_{5} a_{7}^{2}+46 a_{0} a_{1}^{3} a_{2}^{2} a_{3} a_{6} a_{7}^{2} \\
& -36 a_{0} a_{1}^{4} a_{2} a_{3} a_{7}^{3}-50 a_{1}^{2} a_{2}^{4} a_{3}^{2} a_{4} a_{8}+40 a_{1}^{3} a_{2}^{3} a_{3}^{2} a_{5} a_{8}-a_{0} a_{2}^{6} a_{5}^{2} a_{8} \\
& -32 a_{1}^{4} a_{2}^{2} a_{3}^{2} a_{6} a_{8}+16 a_{0} a_{2}^{6} a_{4} a_{6} a_{8}-12 a_{0} a_{1} a_{2}^{5} a_{5} a_{6} a_{8}+12 a_{0} a_{1}^{2} a_{2}^{4} a_{6}^{2} a_{8} \\
& +16 a_{1}^{5} a_{2} a_{3}^{2} a_{7} a_{8}-40 a_{0} a_{1} a_{2}^{5} a_{4} a_{7} a_{8}+34 a_{0} a_{1}^{2} a_{2}^{4} a_{5} a_{7} a_{8} \\
& \left.\left.-44 a_{0} a_{1}^{3} a_{2}^{3} a_{6} a_{7} a_{8}+30 a_{0} a_{1}^{4} a_{2}^{2} a_{7}^{2} a_{8}+8 a_{1}^{5} a_{2}^{2} a_{3} a_{8}^{2}\right)+\mathcal{O}\left(e_{0}^{8}\right)\right) \text {. }
\end{aligned}
$$

Obviously, there is an SU(5) singularity along the GUT divisor defined by $e_{0}=0$. Additionally, if $a_{0}$ has zeros, there will be a further $\mathrm{SU}(2)$ singularity whose Cartan divisor is precisely the divisor obtained from blowing up the $\mathbb{Z}_{2}$ orbifold singularity of $\mathbb{P}_{112}$. Here we ignore this additional part by making sure later on that $a_{0}$ is in fact a constant, which implies that the Calabi-Yau hypersurface avoids the orbifold singularity. Furthermore, there are three different curves on the GUT divisor over which the SU(5) singularity is enhanced, namely

$$
\begin{aligned}
T & \equiv a_{2}=0 \\
F_{1} & \equiv-a_{2}^{3} a_{4}+a_{1} a_{2}^{2} a_{5}-a_{1}^{2} a_{2} a_{6}+a_{1}^{3} a_{7}=0 \\
F_{2} & \equiv-a_{3} a_{7}+a_{2} a_{8}=0 .
\end{aligned}
$$

Since we have that

$$
\begin{aligned}
\left.f\right|_{T=0} & =\mathcal{O}\left(e_{0}^{2}\right), & \left.g\right|_{T=0} & =\mathcal{O}\left(e_{0}^{3}\right), & \left.\Delta\right|_{T=0} & =\mathcal{O}\left(e_{0}^{7}\right) \\
\left.f\right|_{F_{1}=0} & =\mathcal{O}\left(e_{0}^{0}\right), & \left.g\right|_{F_{1}=0} & =\mathcal{O}\left(e_{0}^{0}\right), & \left.\Delta\right|_{F_{1}=0} & =\mathcal{O}\left(e_{0}^{6}\right) \\
\left.f\right|_{F_{2}=0} & =\mathcal{O}\left(e_{0}^{0}\right), & \left.g\right|_{F_{2}=0} & =\mathcal{O}\left(e_{0}^{0}\right), & \left.\Delta\right|_{F_{2}=0} & =\mathcal{O}\left(e_{0}^{6}\right)
\end{aligned}
$$




\begin{tabular}{|cccc|c|}
\hline Equation & Involved curves & Singularity & Coupling & Multiplicity \\
\hline$\left\{a_{1}=0\right\} \cap\left\{a_{2}=0\right\}$ & $T, F_{1}$ & non-minimal & - & 0 \\
$\left\{a_{2}=0\right\} \cap\left\{a_{3}=0\right\}$ & $T, F_{2}$ & $E_{6}$ & $\mathbf{1 0} \times \mathbf{1 0} \times \mathbf{5}^{\prime \prime}$ & 27 \\
$\left\{a_{2}=0\right\} \cap\left\{a_{7}=0\right\}$ & $T, F_{1}, F_{2}$ & $S O(12)$ & $\mathbf{1 0} \times \overline{\mathbf{5}}^{\prime} \times \overline{\mathbf{5}}^{\prime \prime}$ & 18 \\
\hline
\end{tabular}

Table 1. Yukawa couplings involving only non-Abelian representations. Note that all the couplings are located on the GUT divisor defined by $e_{0}=0$. The multiplicities were evaluated explicitly for the example manifold given in subsection 3.5.

\begin{tabular}{|ccc|c|}
\hline Involved curves & Singularity & Coupling & Multiplicity \\
\hline$F_{1}, F_{2}$ & $\mathrm{SU}(7)$ & $\mathbf{1} \times \mathbf{5}^{\prime} \times \overline{\mathbf{5}}^{\prime \prime}$ & 108 \\
\hline
\end{tabular}

Table 2. Yukawa couplings involving both non-Abelian and Abelian representations. Note that all the couplings are located on the GUT divisor defined by $e_{0}=0$. The multiplicities were evaluated explicitly for the example manifold given in subsection 3.5.

we find that there are $\mathrm{SU}(6)$ singularities along the curves $F_{i}=0$ and that there is an $S O(10)$ singularity at $T=0$. Consequently, the $F_{i}=0$ curves host fundamental matter representations, while the $T=0$ curve is the location of the antisymmetric 10 representation of $\mathrm{SU}(5)$. We denote the representation located at $F_{1}=0$ and $F_{2}$ by $\mathbf{5}^{\prime}$ and $5^{\prime \prime}$, respectively.

Before proceeding any further, let us remark here already that without further gauge symmetries than SU(5), one would not expect to find different 5 -curves as we just have. We therefore expect there to be an additional gauge symmetry that can differentiate the two curves. However, from the absence of sections we know that it cannot be an Abelian gauge group. It will, in fact, turn out to be a discrete symmetry that distinguishes the $\mathbf{5}$-curves.

Next, let us consider the Yukawa points on the GUT divisor, i.e. those points at which several of the curves meet and the singularity is enhanced even further. We first consider points that involve the $\mathbf{1 0}$ representation. Since we have that

$$
\begin{aligned}
\left.f\right|_{T=0}=-\frac{1}{3} \cdot( & e_{0}^{2} \cdot a_{1}^{2} \cdot a_{3}^{2}-e_{0}^{3} \cdot a_{0} \cdot a_{1} \cdot\left(a_{3} a_{6}+3 a_{1} a_{8}\right) \\
& \left.+e_{0}^{4} \cdot a_{0} \cdot\left(-3 a_{3}^{2} a_{4}+a_{0} a_{6}^{2}-3 a_{0} a_{5} a_{7}\right)+12 e_{0}^{5} \cdot a_{0}^{2} a_{4} a_{8}\right) \\
\left.g\right|_{T=0}=\frac{1}{864} \cdot( & -64 e_{0}^{3} \cdot a_{1}^{3} \cdot a_{3}^{3}+24 e_{0}^{4} \cdot a_{0} \cdot a_{1}^{2} \cdot\left(4 a_{3}^{2} a_{6}+9 a_{0}^{2} a_{7}^{2}+12 a_{0} a_{1} a_{3} a_{8}\right) \\
& \left.+48 e_{0}^{5} \cdot a_{0} \cdot a_{1} \cdot\left(6 a_{3}^{3} a_{4}+2 a_{0} a_{3} a_{6}^{2}-3 a_{0} a_{3} a_{5} a_{7}-12 a_{0} a_{1} a_{6} a_{8}\right)+\mathcal{O}\left(e_{0}^{6}\right)\right)
\end{aligned}
$$

we find the enhancements listed in table 1.

Additionally, there are couplings between the two 5-curves and singlets under the nonAbelian gauge group. We do not give the explicit equation of the singlet curve here, but note that we find the couplings list in table 2 . 


\begin{tabular}{|cccc|c|}
\hline Equation & Involved curves & Singularity & Coupling & Multiplicity \\
\hline$\left\{a_{1}=0\right\} \cap\left\{a_{2}=0\right\}$ & $T, F_{1}$ & non-minimal & - & 0 \\
$\left\{a_{2}=0\right\} \cap\left\{a_{3}=0\right\}$ & $T, F_{2,1}$ & $E_{6}$ & $\mathbf{1 0} \times \mathbf{1 0} \times \mathbf{5}^{\prime \prime}$ & 27 \\
$\left\{a_{2}=0\right\} \cap\left\{a_{7}=0\right\}$ & $T, F_{1}, F_{2,2}$ & $S O(12)$ & $\mathbf{1 0} \times \overline{\mathbf{5}}^{\prime} \times \overline{\mathbf{5}}^{\prime \prime \prime}$ & 18 \\
\hline
\end{tabular}

Table 3. Yukawa couplings involving only non-Abelian representations. Note that all the couplings are located on the GUT divisor defined by $e_{0}=0$. The multiplicities were evaluated explicitly for the example manifold given in subsection 3.5 after transitioning to the conifold point and resolving the singularities appearing there.

\begin{tabular}{|cccc|c|}
\hline Equation & Involved curves & Singularity & Coupling & Multiplicity \\
\hline- & $F_{1}, F_{2,1}$ & $\mathrm{SU}(7)$ & $\mathbf{1} \times \mathbf{5}^{\prime} \times \overline{\mathbf{5}}^{\prime \prime}$ & 54 \\
- & $F_{1}, F_{2,2}$ & $\mathrm{SU}(7)$ & $\mathbf{1} \times \mathbf{5}^{\prime} \times \overline{\mathbf{5}}^{\prime \prime \prime}$ & 54 \\
$\left\{a_{3}=0\right\} \cap\left\{a_{7}=0\right\}$ & $F_{2,1}, F_{2,2}$ & $\mathrm{SU}(7)$ & $\mathbf{1} \times \mathbf{5}^{\prime \prime} \times \overline{\mathbf{5}}^{\prime \prime \prime}$ & 54 \\
\hline
\end{tabular}

Table 4. Yukawa couplings involving both non-Abelian and Abelian representations. Note that all the couplings are located on the GUT divisor defined by $e_{0}=0$. The multiplicities were evaluated explicitly for the example manifold given in subsection 3.5 after transitioning to the conifold point and resolving the singularities appearing there.

\subsection{Curve splitting and conifold transition}

Before going into the details of the particular base we used in order to compute the precise number of Yukawa points given in the above tables, let us first, in the spirit of [19], go to the conifold locus in moduli space, where we obtain a model with two sections, or equivalently an extra massless U(1). This gives a curve of conifold singularities located at $a_{3}=a_{7}=0$. As noted above, this corresponds to tuning $a_{8} \rightarrow 0$. Interestingly, this transition has an effect on the $\mathbf{5}$-curves in the geometry, since $F_{2}$ becomes reducible:

$$
\left.F_{2}\right|_{a_{8}=0}=-\underbrace{a_{3}}_{F_{2,1}} \cdot \underbrace{a_{7}}_{F_{2,2}}
$$

If we denote the fundamentals at $F_{2,1}=0$ by $\mathbf{5}^{\prime \prime}$ and those at $F_{2,2}=0$ by $\mathbf{5}^{\prime \prime \prime}$ then we find the Yukawa couplings listed in table 3.

Furthermore, in table 4 we summarize the couplings that do not involve the antisymmetric representation.

We do not give explicit expressions for the singlet curve involved in the first two couplings, as they are not complete intersections and contain a large number of terms.

At the conifold locus in complex structure moduli space, we can also compute the $\mathrm{U}(1)$ charges of the matter states using well-known techniques [14]. After rescaling the $\mathrm{U}(1)$ factor to avoid fractional charges, we find the following charge assignments:

$$
\mathbf{1 0}=\mathbf{1 0}_{3}, \quad \mathbf{5}^{\prime}=\mathbf{5}_{-1}, \quad \mathbf{5}^{\prime \prime}=\mathbf{5}_{-6}, \quad \mathbf{5}^{\prime \prime \prime}=\mathbf{5}_{4}
$$


Furthermore, we find that the singlet involved in the $1 \times 5^{\prime \prime} \times \mathbf{5}^{\prime \prime \prime}$ coupling has $\mathrm{U}(1)$-charge 10, while the singlets in the other two $\mathbf{5} \times \overline{\mathbf{5}}$ couplings have U(1)-charge 5 .

\subsection{Discrete charges and forbidden Yukawa couplings}

Finally, let us move away from the conifold locus again by deforming $\tilde{a}_{8} \neq 0$. Looking at the multiplicities of the Yukawa couplings given in tables 1,2, 3, and 4, the following picture about the physics of the deformation process suggests itself rather naturally. The action takes place on the $\mathbf{5}^{\prime \prime}=\mathbf{5}_{-6}$ and $\mathbf{5}^{\prime \prime \prime}=\mathbf{5}_{4}$ curves, since they have the same $\mathbb{Z}_{2}$ charge according to (2.23). We observe that precisely where these two curves intersect, they have a Yukawa coupling with the $\mathbf{1}_{10}$ singlet parameterizing the deformation. As this singlet gets a VEV, the two curves recombine into a single object that we called $\mathbf{5}^{\prime \prime}$ in section 3.2. Since this is a local operation close to the intersection of the two curves, we expect the rest of the Yukawa couplings involving the $\mathbf{1}_{5}$ singlets to simply come along for the ride. And indeed, the multiplicities of the Yukawa points are conserved, if one compares with the results in the previous section.

To finish this subsection, let us quickly summarize the $\mathbb{Z}_{2}$ charges of the matter curves away from the conifold locus. There one finds that ${ }^{8}$

$$
Q_{\mathbb{Z}_{2}}\left(\mathbf{5}^{\prime}\right)=1, \quad Q_{\mathbb{Z}_{2}}\left(\mathbf{5}^{\prime \prime}\right)=0, \quad Q_{\mathbb{Z}_{2}}(\mathbf{1 0})=1,
$$

which is compatible with the couplings we found in table 1 . Note that this is precisely what we expect based on the discussion of section 2.3. In particular, we find that the coupling

$$
10 \times 10 \times 5^{\prime}
$$

is not invariant under the $\mathbb{Z}_{2}$ action and is not realized geometrically, although it would be allowed by all massless continuous symmetries.

\subsection{An explicit example without non-minimal singularities}

After keeping much of the previous discussion independent of the actual choice of base manifold, let us now present the toric data of an explicit example here. In doing this, it is important to recall that as soon as one considers three-dimensional base manifolds, there will generally be non-minimal singularities corresponding to non-flat points of the fibration [16]. We took this into account in the above discussion, making tables 1 and 3 both contain an entry corresponding to such a non-minimal singularity. The relevant conditions will generically have non-trivial solutions at codimension three in the base manifold. The fact that there generically are such non-flat points does not imply that examples without them are impossible, or particularly convoluted. The condition one needs to satisfy is

$$
\left\{a_{1}=0\right\} \cap\left\{a_{2}=0\right\}=\emptyset
$$

and as we will now show some simple geometries admit solutions to this equation.

Our explicit model is as follows. Take a toric ambient space defined by a fine star triangulation of the rays given in table 5 . As can be seen from the defining data, the generic ambient fiber space is $\mathbb{P}_{112}$.

\footnotetext{
${ }^{8}$ Note that since we are not at the conifold locus anymore, $5^{\prime \prime}$ corresponds to the matter curve $F_{2}=0$.
} 


\begin{tabular}{|ccc|cc|cccc|ccc|}
\hline$u_{0}$ & $u_{1}$ & $u_{2}$ & $v_{1}$ & $e_{0}$ & $e_{1}$ & $e_{2}$ & $e_{3}$ & $e_{4}$ & $y_{2}$ & $y_{1}$ & $w$ \\
\hline-3 & 0 & 0 & 0 & 0 & -1 & -2 & -2 & -1 & -1 & -1 & 1 \\
-3 & 0 & 0 & -1 & 0 & 1 & 1 & 0 & 0 & -1 & 1 & 0 \\
0 & 0 & 0 & -1 & 1 & 1 & 1 & 1 & 1 & 0 & 0 & 0 \\
1 & 0 & -1 & 0 & 0 & 0 & 0 & 0 & 0 & 0 & 0 & 0 \\
0 & 1 & -1 & 0 & 0 & 0 & 0 & 0 & 0 & 0 & 0 & 0 \\
\hline
\end{tabular}

Table 5. Homogeneous coordinates of the ambient toric space and the corresponding rays of the toric fan.

The base manifold is $\mathbb{P}^{1} \times \mathbb{P}^{2}$ and the resolved SU(5) singularity discussed in subsection 3.1 lies on the base divisor $\{\mathrm{pt}\} \times \mathbb{P}^{2} \subset \mathbb{P}^{1} \times \mathbb{P}^{2}$. Note that making the geometric transition by going to the conifold locus and resolving the conifold singularities corresponds torically to introducing another ray with entries $(0,1,0,0,0)$ as in [19], which automatically imposes $a_{8}=0$.

Given the explicit data of the ambient space in which our Calabi-Yau manifold is embedded, there is an easy way of confirming the absence of non-flat points. As discussed in [16], at the non-flat points one of the irreducible fiber components grows an extra dimension. In the notation of table 5 , the irreducible fiber components are the horizontal parts of the exceptional divisors $e_{i}=0$. The irreducible fiber component which generically jumps in dimension is the one whose ray does not correspond to a vertex of the top, i.e. $e_{4}=0$.

Let us therefore examine this component with care. On the divisor $e_{4}=0$ the hypersurface equation (3.1) reduces to

$$
\left.p_{112}\right|_{e_{4}=0}=\tilde{a}_{1} \cdot y_{1}^{2} w+\tilde{a}_{2} \cdot y_{2}^{2} w .
$$

However, for the above choice of space, one finds that

$$
\tilde{a}_{1}=e_{1} e_{2} \cdot \underbrace{\left(\alpha_{1} e_{0}+\alpha_{2} v_{1}\right)}_{a_{1}},
$$

with $\alpha_{i}$ two generically non-zero constants. In the base, $e_{0}$ and $v_{1}$ are just the homogeneous coordinates of a $\mathbb{P}_{1}$ and in particular $e_{0}=v_{1}=0$ is forbidden. As a consequence, there are no solutions to $e_{0}=a_{1}=0$.

\section{Conclusions}

In this paper we studied the physical implications of the presence of geometrically massive $\mathrm{U}(1)$ gauge fields in F-theory compactifications without section. F-theory on a genus-one fibered Calabi-Yau fourfold $\mathcal{X}_{4}$ yields a four-dimensional $\mathcal{N}=1$ effective theory that can admit an SU(5) GUT group upon engineering appropriate singularities of the fibration. We considered the case in which $\mathcal{X}_{4}$ does not admit a section, but rather a bi-section. This implies that the fourfold cannot be brought into Weierstrass form, but we showed that 
an SU(5) non-Abelian gauge symmetry can be explicitly implemented. The absence of a section was argued to correspond to the presence of a massive $U(1)$ under which the matter states of the GUT are charged. This imposes stringent condition on the allowed Yukawa couplings, which we analyzed in detail for a specific example.

We provided two perspectives on the massive U(1) gauge field. Firstly, we discussed a closed string perspective, where the $\mathrm{U}(1)$ becomes massive by 'eating' a closed string axion. This axion arises from the R-R or NS-NS two-form in F-theory and the Stückelberg gauging is dependent purely on the geometry of the seven-brane configuration. A dual open string interpretation was given by introducing GUT singlets that carry U(1) charge. Geometrically, these singlets are most naturally identified at special loci in the complex structure moduli space of the Calabi-Yau fourfold at which a curve of conifold singularities is generated. At these loci in moduli space the $U(1)$ is massless and the spectrum of the four-dimensional theory can be extracted using the techniques developed for F-theory compactifications with multiple U(1)s [9, 14, 30]. Moving away from the singular locus can be interpreted as a Higgsing of certain GUT singlets in the open string picture, which corresponds to a recombination of seven-branes in F-theory. We also found that geometrically a recombination of certain $\mathbf{5}$ matter curves occurs in this transition. Such behavior is consistent with discrete selection rules imposed by the now massive U(1) after integrating them out.

The study of Yukawa couplings has revealed that even when restricting to massless modes only, the allowed couplings are restricted by discrete selection rules. In the open string picture this is due to the well-known fact that after Higgs mechanism only a discrete symmetry remains. This also implies that the triple couplings in the superpotential involving the Higgsed singlets turn into mass terms, corresponding precisely to the fact that some of the $\mathbf{5}$ matter curves recombine in the Higgs branch. Remarkably, the closed string interpretation of the couplings involving the Higgsed singlets requires the presence of new instanton effects that are not suppressed by a volume modulus. The precise interpretation of the instanton effects in F-theory or its weak string coupling Type IIB limit is still lacking and would be of importance. In M-theory the non-perturbative effects arise from M2-branes wrapped on the shrinking 3-spheres along the conifold curve. We argued that this geometric picture allows to identify the key ingredients of the field theory setup including the massive $\mathrm{U}(1)$ arising from the expansion into non-closed forms. Clearly, it would be interesting to complete this picture further elucidating the Yukawa couplings and their relation to T-branes.

\section{Acknowledgments}

We would like to thank Lara Anderson, Volker Braun, and Diego Regalado for illuminating discussions. I.G.-E. thanks N. Hasegawa for kind encouragement and constant support.

Open Access. This article is distributed under the terms of the Creative Commons Attribution License (CC-BY 4.0), which permits any use, distribution and reproduction in any medium, provided the original author(s) and source are credited. 


\section{References}

[1] C. Vafa, Evidence for F-theory, Nucl. Phys. B 469 (1996) 403 [hep-th/9602022] [INSPIRE].

[2] N. Nakayama, On Weierstrass models, in Algebraic geometry and commutative algebra. In honor of M. Nagata, M. Nagata and H. Hijikata eds., Academic Press, New York, (1987).

[3] A. Grassi and D.R. Morrison, Anomalies and the Euler characteristic of elliptic Calabi-Yau threefolds, Commun. Num. Theor. Phys. 6 (2012) 51 [arXiv:1109.0042] [INSPIRE].

[4] T.W. Grimm and T. Weigand, On abelian gauge symmetries and proton decay in global F-theory GUTs, Phys. Rev. D 82 (2010) 086009 [arXiv:1006.0226] [InSPIRE].

[5] P. Deligne, Courbes Elliptiques: formulaire d'apres J. Tate, in Modular functions of one variable, IV, B.J. Birch and W. Kuyk eds., Lecture notes in mathematics volume 476, Springer, Germany (1975).

[6] D.R. Morrison and D.S. Park, F-theory and the Mordell-Weil group of elliptically-fibered Calabi-Yau threefolds, JHEP 10 (2012) 128 [arXiv:1208.2695] [INSPIRE].

[7] J. Borchmann, C. Mayrhofer, E. Palti and T. Weigand, Elliptic fibrations for $\mathrm{SU}(5) \times \mathrm{U}(1) \times \mathrm{U}(1)$ F-theory vacua, Phys. Rev. D 88 (2013) 046005 [arXiv: 1303.5054] [INSPIRE].

[8] M. Cvetič, D. Klevers and H. Piragua, F-theory compactifications with multiple U(1)-factors: constructing elliptic fibrations with rational sections, JHEP 06 (2013) 067 [arXiv:1303.6970] [INSPIRE].

[9] J. Borchmann, C. Mayrhofer, E. Palti and T. Weigand, SU(5) tops with multiple U(1)s in F-theory, Nucl. Phys. B 882 (2014) 1 [arXiv:1307.2902] [InSPIRE].

[10] M. Cvetič, D. Klevers and H. Piragua, F-theory compactifications with multiple U(1)-factors: addendum, JHEP 12 (2013) 056 [arXiv: 1307.6425] [INSPIRE].

[11] M. Cvetič, D. Klevers, H. Piragua and P. Song, Elliptic fibrations with rank three Mordell-Weil group: F-theory with $\mathrm{U}(1) \times \mathrm{U}(1) \times \mathrm{U}(1)$ gauge symmetry, JHEP 03 (2014) 021 [arXiv: 1310.0463] [INSPIRE].

[12] C. Mayrhofer, E. Palti and T. Weigand, U(1) symmetries in F-theory GUTs with multiple sections, JHEP 03 (2013) 098 [arXiv:1211.6742] [INSPIRE].

[13] M. Kuntzler and S. Schäfer-Nameki, Tate trees for elliptic fibrations with rank one Mordell-Weil group, arXiv:1406.5174 [INSPIRE].

[14] V. Braun, T.W. Grimm and J. Keitel, New global f-theory GUTs with U(1) symmetries, JHEP 09 (2013) 154 [arXiv:1302.1854] [INSPIRE].

[15] T.W. Grimm, A. Kapfer and J. Keitel, Effective action of $6 D$ F-theory with U(1) factors: rational sections make Chern-Simons terms jump, JHEP 07 (2013) 115 [arXiv:1305.1929] [INSPIRE].

[16] V. Braun, T.W. Grimm and J. Keitel, Geometric engineering in toric F-theory and GUTs with U(1) gauge factors, JHEP 12 (2013) 069 [arXiv: 1306.0577] [INSPIRE].

[17] V. Braun and D.R. Morrison, F-theory on genus-one fibrations, JHEP 08 (2014) 132 [arXiv:1401.7844] [INSPIRE].

[18] D.R. Morrison and W. Taylor, Sections, multisections and U(1) fields in F-theory, arXiv: 1404.1527 [INSPIRE]. 
[19] L.B. Anderson, I. García-Etxebarria, T.W. Grimm and J. Keitel, Physics of F-theory compactifications without section, arXiv:1406.5180 [INSPIRE].

[20] D. Klevers, D.K. Mayorga Pena, P.-K. Oehlmann, H. Piragua and J. Reuter, F-Theory on all toric hypersurface fibrations and its Higgs branches, arXiv:1408.4808 [INSPIRE].

[21] V. Braun, Toric elliptic fibrations and F-theory compactifications, JHEP 01 (2013) 016 [arXiv:1110.4883] [INSPIRE].

[22] S. Krippendorf, D.K. Mayorga Pena, P.-K. Oehlmann and F. Ruehle, Rational F-theory GUTs without exotics, JHEP 07 (2014) 013 [arXiv: 1401.5084] [INSPIRE].

[23] C. Lawrie and S. Schäfer-Nameki, The Tate form on steroids: resolution and higher codimension fibers, JHEP 04 (2013) 061 [arXiv:1212.2949] [INSPIRE].

[24] H. Hayashi, C. Lawrie and S. Schäfer-Nameki, Phases, flops and F-theory: SU(5) gauge theories, JHEP 10 (2013) 046 [arXiv: 1304.1678] [INSPIRE].

[25] H. Hayashi, C. Lawrie, D.R. Morrison and S. Schäfer-Nameki, Box graphs and singular fibers, JHEP 05 (2014) 048 [arXiv: 1402.2653] [INSPIRE].

[26] A.P. Braun and S. Schäfer-Nameki, Box graphs and resolutions I, arXiv:1407.3520 [INSPIRE].

[27] A. Grassi, J. Halverson and J.L. Shaneson, Matter from geometry without resolution, JHEP 10 (2013) 205 [arXiv:1306 .1832] [INSPIRE].

[28] A. Grassi, J. Halverson and J.L. Shaneson, Non-abelian gauge symmetry and the Higgs mechanism in F-theory, arXiv: 1402.5962 [INSPIRE].

[29] S. Krause, C. Mayrhofer and T. Weigand, $G_{4}$ flux, chiral matter and singularity resolution in F-theory compactifications, Nucl. Phys. B 858 (2012) 1 [arXiv:1109.3454] [InSPIRE].

[30] M. Cvetič, A. Grassi, D. Klevers and H. Piragua, Chiral four-dimensional f-theory compactifications with SU(5) and multiple U(1)-factors, JHEP 04 (2014) 010 [arXiv: 1306.3987] [INSPIRE].

[31] T.W. Grimm and H. Hayashi, F-theory fluxes, chirality and Chern-Simons theories, JHEP 03 (2012) 027 [arXiv:1111.1232] [inSPIRE].

[32] M. Cvetič, T.W. Grimm and D. Klevers, Anomaly cancellation and abelian gauge symmetries in F-theory, JHEP 02 (2013) 101 [arXiv:1210.6034] [INSPIRE].

[33] M. Bies, C. Mayrhofer, C. Pehle and T. Weigand, Chow groups, Deligne cohomology and massless matter in F-theory, arXiv:1402.5144 [INSPIRE].

[34] L. Lin and T. Weigand, Towards the standard model in F-theory, arXiv:1406.6071 [INSPIRE].

[35] S. Cecotti, C. Cordova, J.J. Heckman and C. Vafa, T-branes and monodromy, JHEP 07 (2011) 030 [arXiv:1010.5780] [INSPIRE].

[36] L.B. Anderson, J.J. Heckman and S. Katz, T-branes and geometry, JHEP 05 (2014) 080 [arXiv: 1310.1931] [INSPIRE].

[37] H. Hayashi, T. Kawano, R. Tatar and T. Watari, Codimension-3 singularities and Yukawa couplings in F-theory, Nucl. Phys. B 823 (2009) 47 [arXiv:0901.4941] [INSPIRE].

[38] A. Font and L.E. Ibáñez, Matter wave functions and Yukawa couplings in F-theory grand unification, JHEP 09 (2009) 036 [arXiv:0907.4895] [INSPIRE]. 
[39] L. Aparicio, A. Font, L.E. Ibáñez and F. Marchesano, Flux and instanton effects in local F-theory models and hierarchical fermion masses, JHEP 08 (2011) 152 [arXiv:1104.2609] [INSPIRE].

[40] J. Marsano and S. Schäfer-Nameki, Yukawas, G-flux and spectral covers from resolved Calabi-Yau's, JHEP 11 (2011) 098 [arXiv:1108.1794] [INSPIRE].

[41] A. Font, L.E. Ibáñez, F. Marchesano and D. Regalado, Non-perturbative effects and Yukawa hierarchies in F-theory SU(5) Unification, JHEP 03 (2013) 140 [Erratum ibid. 1307 (2013) 036] [arXiv: 1211.6529] [INSPIRE].

[42] A. Font, F. Marchesano, D. Regalado and G. Zoccarato, Up-type quark masses in SU(5) F-theory models, JHEP 11 (2013) 125 [arXiv: 1307.8089] [INSPIRE].

[43] P. Berglund, J.R. Ellis, A.E. Faraggi, D.V. Nanopoulos and Z. Qiu, Elevating the free fermion $Z_{2} \times Z_{2}$ orbifold model to a compactification of F-theory, Int. J. Mod. Phys. A 15 (2000) 1345 [hep-th/9812141] [INSPIRE].

[44] J. de Boer et al., Triples, fluxes and strings, Adv. Theor. Math. Phys. 4 (2002) 995 [hep-th/0103170] [INSPIRE].

[45] M. Berasaluce-Gonzalez, L.E. Ibáñez, P. Soler and A.M. Uranga, Discrete gauge symmetries in D-brane models, JHEP 12 (2011) 113 [arXiv:1106.4169] [INSPIRE].

[46] P. Anastasopoulos, M. Cvetič, R. Richter and P.K.S. Vaudrevange, String constraints on discrete symmetries in MSSM type II quivers, JHEP 03 (2013) 011 [arXiv:1211.1017] [INSPIRE].

[47] G. Honecker and W. Staessens, To tilt or not to tilt: discrete gauge symmetries in global intersecting D-brane models, JHEP 10 (2013) 146 [arXiv:1303.4415] [INSPIRE].

[48] T.W. Grimm, M. Kerstan, E. Palti and T. Weigand, Massive abelian gauge symmetries and fluxes in F-theory, JHEP 12 (2011) 004 [arXiv:1107.3842] [INSPIRE].

[49] A.P. Braun, A. Collinucci and R. Valandro, The fate of U(1)'s at strong coupling in F-theory, JHEP 07 (2014) 028 [arXiv: 1402.4054] [INSPIRE].

[50] H. Jockers and J. Louis, The effective action of D7-branes in $N=1$ Calabi-Yau orientifolds, Nucl. Phys. B 705 (2005) 167 [hep-th/0409098] [INSPIRE].

[51] E. Witten, Nonperturbative superpotentials in string theory, Nucl. Phys. B 474 (1996) 343 [hep-th/9604030] [INSPIRE].

[52] M. Haack and J. Louis, Duality in heterotic vacua with four supercharges, Nucl. Phys. B 575 (2000) 107 [hep-th/9912181] [INSPIRE].

[53] T.W. Grimm, The $N=1$ effective action of F-theory compactifications, Nucl. Phys. B 845 (2011) 48 [arXiv:1008.4133] [InSPIRE].

[54] T.W. Grimm, Axion inflation in F-theory, Phys. Lett. B 739 (2014) 201 [arXiv:1404.4268] [INSPIRE].

[55] R. Blumenhagen, M. Cvetič, S. Kachru and T. Weigand, D-brane instantons in type II orientifolds, Ann. Rev. Nucl. Part. Sci. 59 (2009) 269 [arXiv:0902.3251] [inSPIRE].

[56] A. Strominger, Massless black holes and conifolds in string theory, Nucl. Phys. B 451 (1995) 96 [hep-th/9504090] [InSPIRE].

[57] B.R. Greene, D.R. Morrison and A. Strominger, Black hole condensation and the unification of string vacua, Nucl. Phys. B 451 (1995) 109 [hep-th/9504145] [INSPIRE]. 
[58] B.R. Greene, D.R. Morrison and C. Vafa, A geometric realization of confinement, Nucl. Phys. B 481 (1996) 513 [hep-th/9608039] [INSPIRE].

[59] H. Ooguri and C. Vafa, Summing up D instantons, Phys. Rev. Lett. 77 (1996) 3296 [hep-th/9608079] [INSPIRE].

[60] K. Intriligator, H. Jockers, P. Mayr, D.R. Morrison and M.R. Plesser, Conifold transitions in M-theory on Calabi-Yau fourfolds with background fluxes, Adv. Theor. Math. Phys. 17 (2013) 601 [arXiv:1203.6662] [InSPIRE].

[61] T.W. Grimm, T.-W. Ha, A. Klemm and D. Klevers, Computing brane and flux superpotentials in F-theory compactifications, JHEP 04 (2010) 015 [arXiv:0909.2025] [INSPIRE].

[62] N.C. Bizet, A. Klemm and D.V. Lopes, Landscaping with fluxes and the E8 Yukawa point in F-theory, arXiv:1404.7645 [INSPIRE].

[63] V. Bouchard and H. Skarke, Affine Kac-Moody algebras, CHL strings and the classification of tops, Adv. Theor. Math. Phys. 7 (2003) 205 [hep-th/0303218] [INSPIRE]. 